Received: 2015.06.24 Accepted: 2015.07.09 Published: 2015.08.20

Authors' Contribution: Study Design A Data Collection B Statistical Analysis C Data Interpretation D Manuscript Preparation E Literature Search F Funds Collection G

Corresponding Author: Conflict of interest:

\title{
Idiopathic Bilateral External Jugular Vein Thrombosis
}

Department of Medicine, Hamad Medical Corporation, Doha, Qatar

Patient:

Male, 21

Final Diagnosis:

Symptoms:

Medication:

Idiopathic bilateral external jugular vein thrombosis

Face engorgement $\bullet$ neck swelling

Clinical Procedure:

None

Specialty:

Hematology

Objective:

Background:

Case Report:

\section{Unknown ethiology}

Vein thrombosis is mainly determined by 3 factors, which constitute a triad called Virchow's triad: hypercoagulability, stasis, and endothelial injury. Venous thrombosis commonly occurs in the lower extremities since most of the blood resides there and flows against gravity. The veins of the lower extremities are dependent on intact valves and fully functional leg muscles. However, in case of valvular incompetency or muscular weakness, thrombosis and blood stasis will occur as a result. In contrast, the veins of the neck, specially the jugulars, have distensible walls which allow flexibility during respiration. In addition, the blood directly flows downward towards the heart. Nevertheless, many case reports mentioned the thrombosis of internal jugular veins and external jugular veins with identified risk factors. Jugular vein thrombosis has previously been associated in the literature with a variety of medical conditions, including malignancy.

This report is of a case of idiopathic bilateral external jugular vein thrombosis in a 21 year-old male construction worker of Southeast Asian origin with no previous medical history who presented with bilateral facial puffiness of gradual onset over 1 month. Doppler ultrasound and computed tomography were used in the diagnosis. Further work-up showed no evidence of infection or neoplasia. The patient was eventually discharged on warfarin. The patient was assessed after 6 months and his symptoms had resolved completely.

Conclusions: Bilateral idiopathic external jugular veins thrombosis is extremely rare and can be an indicator of early malignancy or hidden infection. While previous reports in the literature have associated jugular vein thrombosis with malignancy, the present case shows that external jugular vein thrombosis can also be found in persons without malignancy.

MeSH Keywords: Jugular Veins • Venous Thrombosis • Warfarin

Full-text PDF: http://www.amjcaserep.com/abstract/index/idArt/895124

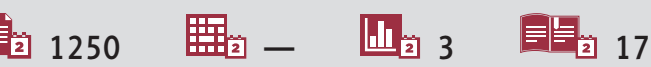




\section{Background}

Venous thrombosis usually results from 3 factors, which constitute a triad called Virchow's triad. These 3 factors are: hypercoagulability, stasis, and endothelial injury [1].

In general, veins of the lower extremities depend on fully competent valves, which prevent retrograde flow and venous hypertension. The muscles of the legs (mainly soleus muscle) also play an important role in the venous system; they pump the blood of the lower limbs and enhance the contractility of the veins. In case of incompetent valves or muscle weakness, blood stasis and thrombosis will eventually occur as a result. Since most of the blood resides in the lower extremities, thrombosis of their veins is considered very common $[2,3]$.

The veins of the neck, specially the jugulars, are more tolerable and resistant to thrombosis than the veins in the lower extremities. They have distensible walls, which facilitates repeated expansion and collapse during respiration. In addition, the direction of drainage of the neck veins is downward with gravity. Thus, thrombosis of neck veins is uncommon $[4,5]$.

Neck veins thrombosis is mostly caused by central venous catheterization, trauma, intravenous drug use, infections, and extrinsic compression by tumors of different locations [3-6]. Taking the internal jugular veins into consideration, the risk factors of thrombosis have been described in a study by Gbaguidi et al. The study categorized the causes into primary and secondary. They labeled cancer, central venous catheter, and ovarian hyper stimulation syndrome as the most common causes of internal jugular vein thrombosis. Other causes include pacemaker insertion, thrombophilia, thoracic outlet syndrome, effort, and idiopathic [7]. Lastly, Lemierre's syndrome is a known cause of thrombosis in internal jugular veins, but is considered uncommon [8].

There are few published reports on external jugular vein thrombosis. Several case reports have described risk factors of external jugular vein thrombosis. Gale et al. reported a case of an elderly male with proximal humeral fracture; the thrombosis of the jugular vein was attributed to external compression by a tight collar and cuff [9]. Another study by Safadi et al., mentioned that neck masses, such as thyroid tumors, can compress the cervical region, which leads to external jugular vein dilatation and thrombosis [10].

Colomina et al. also reported the formation of external jugular veins thrombosis in a patient who had an abdominal surgery, which was most directly related to high intra-abdominal pressure and a supine decubitus Trendelenburg position. Other factors, such as obesity, age, and sex, had a possible role [11]. Aneurysm of the external jugular vein can also predispose to thrombus formation [12]. Lemierre's syndrome has been associated with external jugular vein thrombosis in several case reports $[6,13-15]$.

Lastly, external jugular vein thrombosis can also be idiopathic in rare circumstances and after excluding other conditions. Idiopathic unilateral external jugular vein thrombosis has been reported by Bartella et al. [16]. Sengupta et al. reported a case of bilateral external jugular vein thrombosis that was considered idiopathic after ruling out secondary causes [17]. Considering the rarity of both conditions, we report a case of a young man with bilateral external jugular veins thrombosis.

\section{Case Report}

A 21-year-old southeast Asian male construction worker with no significant past medical history presented with painless bilateral neck swellings associated with facial puffiness and engorgement, which was first noticed by the patient 1 month earlier and reportedly had worsen gradually. There was no history of trauma, cannulation of the neck vessels, weight loss, fever, night sweats, loss of appetite, spontaneous bleeding, chronic pain, shortness of breath, wearing tight neck collar, travel history, exposure to sick contacts, previous similar episodes, previous history of deep vein thrombosis, or family history of similar symptoms. The patient was not taking any medications.

On physical examination, the patient was afebrile, with normal vital signs. The patient's face was plethoric with moderate facial swelling independent of movement of neck or raising the arms. The neck exam showed bilateral cord-like structures on both sides measured about $2 \mathrm{~cm}$ in width extending from the base of the neck to the angle of the mandible. There was no lymphadenopathy. Physical examination of the cardiovascular system, chest, abdomen, central nervous system, genitourinary system was unremarkable.

Initial lab investigations, including complete blood count, basic metabolic panel, liver function tests, and kidney function parameters, were within normal limits. Doppler ultrasound of the neck revealed non-compressible bilateral external jugular veins without a flow, suggesting thrombosis of external jugular veins. The internal jugular veins on both sides were patent (Figures $1 \mathrm{~A}-\mathrm{C}, 2 \mathrm{~A}-\mathrm{C}$ ).

Further investigations were made, including coagulation profile and activity of protein $\mathrm{S}$ and protein $\mathrm{C}$, which were all within normal range. Auto-immune workups, including rheumatoid factor, anti-cyclic citrullinated peptide antibody, anti-nuclear antibody, anti-phospholipids antibodies, and complements (C3 and C4), showed no significance. HIV testing was negative. Inflammatory markers were also sent, including erythrocyte 


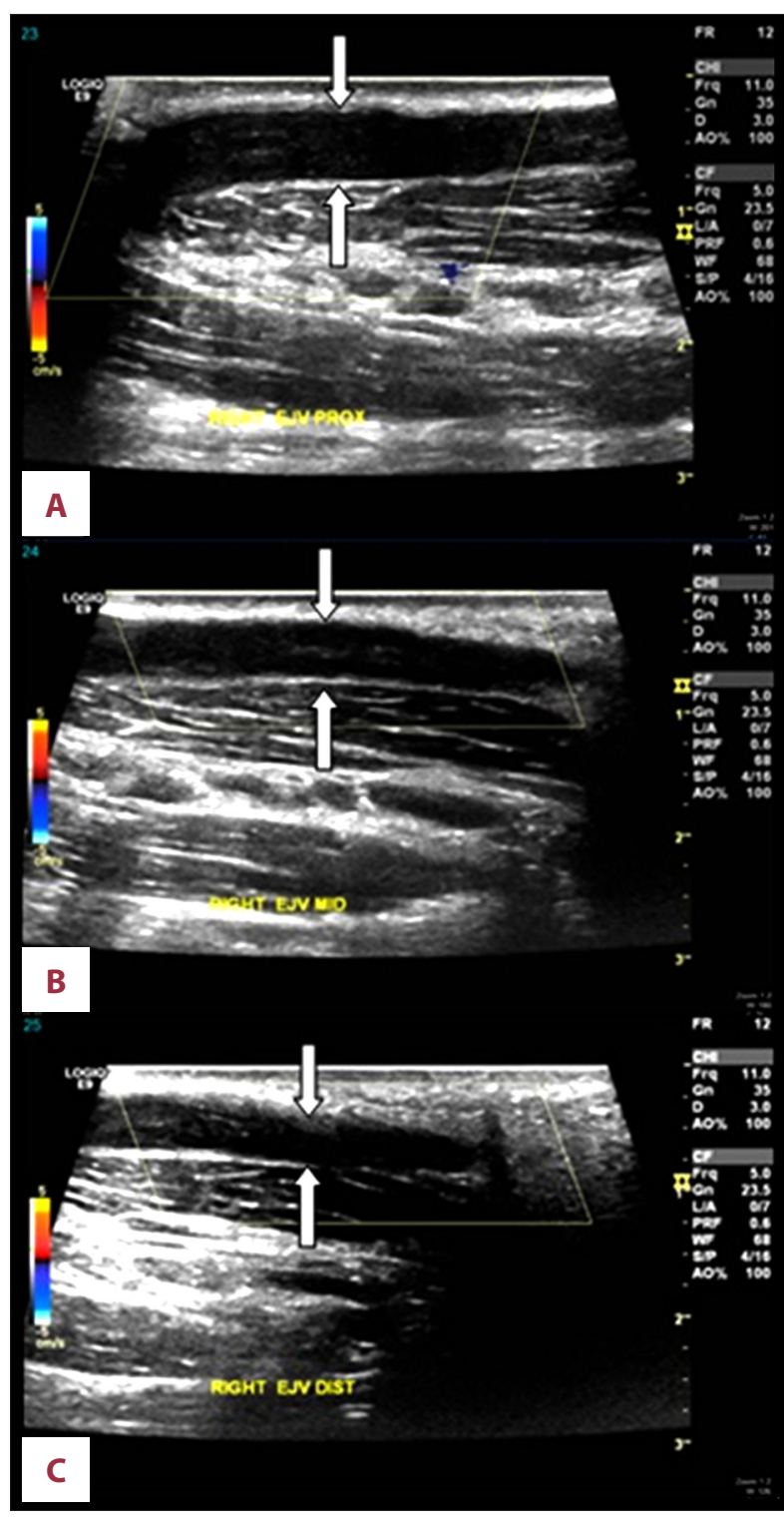

Figure 1. Ultrasound Doppler of the right external jugular vein showing filling defect suggestive of thrombosis. Right proximal external jugular vein (A). Right middle external jugular vein (B). Right distal external jugular vein (C).

sedimentation rate, C-reactive protein, procalcitonin) which all appeared to be within normal limits.

A computed tomography (CT) scan of the neck confirmed the presence of thrombosis in the external jugular veins bilaterally (Figure 3). CT of the chest and abdomen showed no abnormalities. The patient was started on anti-coagulation treatment with heparin initially, and then was kept on warfarin. At the follow-up visit after 6 months, the patient's symptoms had resolved completely and he had developed no other symptoms.

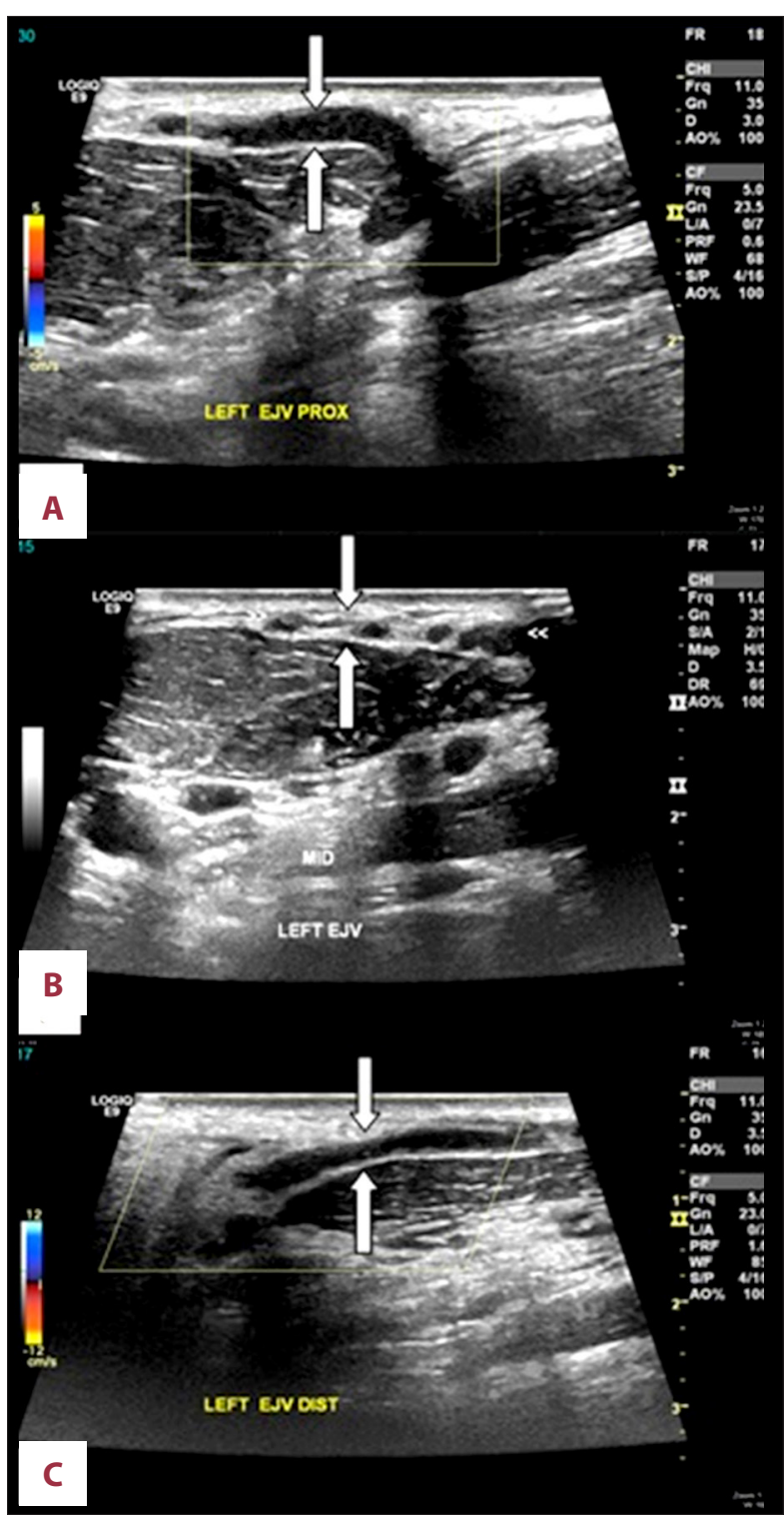

Figure 2. Ultrasound Doppler of the left external jugular vein showing filling defect suggestive of thrombosis. Left proximal external jugular vein (A). Left middle external jugular vein (B). Left distal external jugular vein (C).

\section{Discussion}

After reviewing the literature, we found only 1 prior case report of idiopathic bilateral external jugular veins thrombosis, published by Sengupta et al. They described a 45 -year-old man who presented with facial puffiness and bilateral neck swellings. Two-dimensional echocardiography and Doppler studies showed thrombosis of both the external jugular veins, and digital subtraction angiography (DSA) revealed non-visualization of external jugular veins on both sides. Further work-up was unremarkable. The patient was discharged on aspirin and his symptoms resolved within 1 year after discharge [17]. 


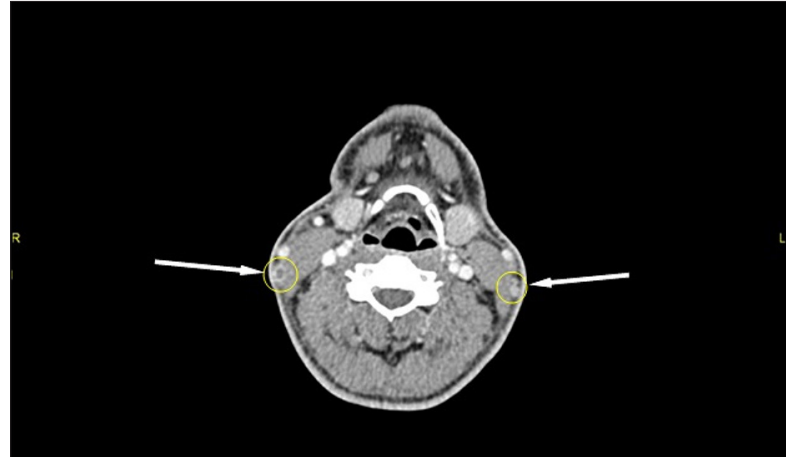

Figure 3. CT scan of the neck showing bilateral external jugular vein thrombosis.

Our case and that reported by Sengupta et al. had similar presentations with no known risk factors, which required extensive work-up. The diagnosis of an idiopathic condition cannot be reached until all other possible causes have been excluded. Thus, a follow-up was arranged and the symptoms in both cases had resolved completely and no other manifestations or focus of malignancy developed. The study by Gbaguidi et al. mentioned similar conditions, in which bilateral internal veins thrombosis was considered as an indicator of malignancy [7].

Most of the reported cases of external jugular vein thrombosis were treated with anticoagulants, which led to complete resolution of the symptoms. The cases caused by Lemierre's syndrome used the combination of antibiotics and anticoagulants, although the use of anticoagulants in Lemierre's is still controversial [6,13-15]. Gale et al. and Colomina et al. both

\section{References:}

1. Dickson BC: Venous thrombosis: on the history of Virchow's triad. Univ Toronto Med J, 2004; 81: 166

2. Meissner MH: Lower extremity venous anatomy. Semin Intervent Radiol, 2005; 22: 147-56

3. Kimura T, Chino M, Ogasawara $\mathrm{N}$ et al: Trousseau's syndrome with brachiocephalic vein thrombosis in a patient with uterine carcinoma. Angiology, 1999; 50: 515-18

4. Liu PG, Jacobs JB, Reede D: Trousseau's syndrome in the head and neck. Am J Otolaryngol, 1985; 6: 405-8

5. Kalan A, Tariq M, Harar RP, Gatland D: Spontaneous internal jugular vein thrombosis and recurrent laryngeal nerve palsy: A rare simultaneous presentation of an occult malignant neoplasm. J Laryngol Otol, 1996; 110: 1166-68

6. Schwartz HC, Nguyen DC: Postanginal septicaemia with external jugular vein thrombosis: Case report. Br J Oral Maxillo Fac Surg, 1999; 37: 144-46

7. Gbaguidi X, Janvresse A, Benichou J et al: Internal jugular vein thrombosis: outcome and RISK factors. QJM, 2011; 104(3): 209-19

8. Karkos PD, Asrani S, Karkos CD et al: Lemierre's syndrome: a systematic review. Laryngoscope, 2009; 119: 1552-59 used anticoagulants [9,11]. This suggests that anticoagulants are essential for symptomatic and preventive treatment of external jugular vein thrombosis.

Regarding the recurrence of external jugular vein thrombosis, there is no published report of any signs of recurrence of thrombosis in external jugular veins $[6,9,11,13-15]$. Moreover, the study by Gbaguidi et al. also reported no recurrence of thrombosis in internal jugular veins in the 29 patients after 12-month follow-up [7].

\section{Conclusions}

Bilateral external jugular veins thrombosis is an extremely rare condition that can occur in individuals with predisposing medical conditions, or it can be idiopathic. Follow-up is important to rule-out any hidden infections or malignancies before concluding that the condition is idiopathic.

\section{Conflict of interests}

The authors have no conflict of interests regarding publishing this paper.

\section{Disclosure statement}

The authors have nothing to disclose. This case report received no specific grant from any funding agency in the public, commercial, or not-for-profit sectors.

9. Gale M, Craxford S, Taylor L et al: Thrombosis of the external jugular vein: Thrombosis of the external jugular vein: a rare complication of a proximal humerus fracture treated with collar and cuff immobilisation. Case Reports in Orthopedics, 2014; 2014: 283790

10. Safadi R, Gafanovich I, Rashkovsky I, Koplewitz B: Isolated external jugular vein thrombosis in older people. J Am Geriatr Soc, 1996; 44: 88-89

11. Colomina MJ, Godet C, Bagó J et al: Isolated thrombosis of the external jugular vein. Surg Laparosc Endosc Percutan Tech, 2000; 10(4):264-67

12. Beale TJ, Smedley FH, Knee G: Thrombosis within an external jugular venous aneurysm. J R Coll Surg Edinb, 1996; 41(3): 181-82

13. Hagiya $\mathrm{H}$, Haruki $\mathrm{Y}$, Otsuka F: Lemierre syndrome involving external jugular vein. Acute Medicine \& Surgery, 2015; 2: 64-68

14. Shibasaki WY, Yoshikawa $\mathrm{H}$, Idezuka J et al: Cerebral infarctions and brain abscess due to Lemierre syndrome. Intern Med, 2005; 44: 653-56

15. Abe H, Kisara A, Yagishita Y: A case of Lemierre syndrome. ICU \& CCU, 1998; 22: 281-85

16. Bartella A, Lübbers HT, Schuknecht B et al: [Thrombosis of the external jug ular vein. Case report of a rare cause for pain in the lateral neck.] Swiss Dent J, 2014; 124(1): 39-48 [in French, German]

17. Sengupta $S$, Kalkonde $Y$, Khot $R$ et al: Idiopathic bilateral external jugular vein thrombosis - a case report. Angiology, 2001; 52(1): 69-71 